Running title: Benign and malignant oral lesions share risk factors.

\title{
Screening for oral cancer utilising risk-factor analysis is ineffective in high-risk populations.
}

Lalli $\mathrm{A}^{1}$, Aldehlawi $\mathrm{H}^{1}$, Buchanan $\mathrm{JAG}^{2}$, Seoudi $\mathrm{N}^{2}$, Fortune $\mathrm{F}^{1}$, Waseem $\mathrm{A}^{1}$.

${ }^{1}$ Centre for Oral Immunobiology and Regenerative Medicine

Institute of Dentistry

Barts and the London School of Medicine and Dentistry

Queen Mary University of London

Newark Street

London E1 2AT

${ }^{2}$ Centre for Teaching Innovation

Institute of Dentistry

Barts and the London School of Medicine and Dentistry

Queen Mary University of London

Newark Street

London E1 2AT

Correspondence to:

Anand Lalli: Office 4, 4th Floor, Institute of Dentistry, Barts and the London School of Medicine and Dentistry, Queen Mary University of London, Newark Street. London E1 2AT. Tel: 02078828618

Fax: 02073777121

Email: a.lalli@qmul.ac.uk 


\section{ABSTRACT}

\section{Objective}

Oral cancer screening by direct visual examination is believed to be ineffective because of the difficulty in differentiating a small number of malignancies from the much more prevalent benign oral mucosal lesions (OMLs) found in high-risk individuals.

\section{Study Design}

Standardised clinical diagnoses were recorded for all OMLs identified during oral visual examination of 1111 individuals displaying risk factors for oral cancer including tobacco and areca nut (paan) consumption. Suspicious lesions were referred for biopsy and definitive diagnosis.

\section{Results}

1438 OMLs with 32 different clinical diagnoses were identified in 604 participants. Analysis of referrals revealed two distinct groups termed: Visually Benign Lesions (VBLs) where none were referred and Visually Complex Lesions (VCLs) comprising 661 OMLs with 9 different clinical diagnosis. After biopsy VCLs were shown to include the known potentially malignant disorders (PMDs) as well as benign lesions such as paan mucositis. VCLs, but not VBLs, share risk factors with oral cancer ( $p<0.05$ for paan $5.82(\mathrm{Cl}: 1.98-$ 8.43) and smoking $3.59(\mathrm{Cl}: 1.12-4.47)$ ).

\section{Conclusion}

VCLs are clinically indistinguishable but much more prevalent than oral cancer and most will never undergo malignant change. Therefore VCLs prevent dentists accurately detecting malignancy during the clinical examination of high-risk patients. However, VCLs can easily be differentiated from other benign OMLs by visual examination alone. Further research into diagnostic technology is needed to help differentiate VCLs from oral cancers. 


\section{INTRODUCTION}

Every dental practitioner has been faced with the problem of whether to observe or intervene when they detect an oral mucosal lesion (OML) because they can represent oral cancer (OSCC) or a potentially malignant disorder (PMD). A comprehensive review of the accuracy of oral examination as a screening test for oral cancer found sensitivity estimates varying from 0.50 (95\% $\mathrm{Cl} 0.07$ to 0.93$)$ to $0.99(95 \% \mathrm{Cl} 0.97 \text { to } 1.00)^{1}$. This suggests clinicians observing OMLs may only be correct in suspecting malignancy about half the time, confirming that clinical presentations of OSCCs aren't always easily distinguishable from benign OMLs. Brocklehurst et. al. provided further evidence for this by surveying dentists for their cues to referral of suspected malignancy and found most related to advanced lesions (induration, ragged borders, fixation) and patient risk factors (smoking $>20$ cigarettes/day) and concluded that early OSCCs and PMDs could easily be missed ${ }^{2}$. The role of risk factors in the decision making process hasn't previously been evaluated but would be a significant issue if benign OMLs shared risk factors with OSCC in known high-risk individuals such as tobacco or paan users. In this study we evaluate the relationship between OSCC risk factors and the decision to refer for suspected malignancy during screening of a high-risk South Asian origin population of the UK. 


\section{METHODS}

Development of a targeted opportunistic screening protocol for the high-risk South Asian origin population of East London, has been reported previously ${ }^{3}$. A population-based survey was integrated to record the prevalence of OMLs in those screened for OSCC.

The clinical diagnosis of all OMLs observed was recorded whether referred or not. Standardisation was aided by developing a defined list of OML clinical diagnosis based on the accepted nomenclature from Warnakulasuriya et. al. ${ }^{4}$ and the list utilised by Pearson et. al. ${ }^{5}$ in their study of a similar UK population (Table 1). Other lesions and conditions were as described in Cawson's Essentials of Oral Pathology and Oral Medicine $7^{\text {th }}$ Edition (2002) ${ }^{6}$. OSCCs predominantly develop at specific sites in the mouth so it is believed that OMLs at different sites carry different risks of malignant conversion ${ }^{7}$. Therefore, the site of lesions was recorded on a mouth map. All patient screened for OSCC were asked about their risk habits, including smoking and areca nut extract usage or alcohol consumption. In view of the opportunistic screening methodology employed, it was not possible to followup individuals screened negative.

Data analysis utilised Excel 2003 (Microsoft, USA) with the StatPro add-on (Kelley School of Business, Indiana University) and STATA 10 (StataCorp LP, Texas, USA). 


\section{RESULTS}

In total 1438 OMLs and 32 different clinical diagnosis were observed in 604 individuals in the screened population of 1111. In total, 66 were screened positive with 2 histologically confirmed oral cancers and 10 dysplastic lesions.

\section{Characteristics of Oral Mucosal Lesions Detected}

Frictional keratosis and paan staining were by far the commonest lesions detected, comprising 522 (36\%) and 520 (36\%) of all OMLs respectively (Table 1).

Sixty leukoplakias were reported, representing $4.1 \%$ of all OMLs. This is considerably lower than that reported by Pearson et. al. ${ }^{5}$ in a similar population of the UK and more consistent with the later trials reported in 2006 and 2011 in India ${ }^{8,9}$. This may in part be due to the updating of the definition of leukoplakia in the interim ${ }^{4}$ so some clinically detectable white patches previously called leukoplakia may have been further classified by cause, for example, as frictional keratosis. Some evidence of this comes from the fact that 32 different lesions were reported in our study whilst Pearson et. al. ${ }^{5}$ reported just 13 .

\section{Referred and Screened Oral Mucosal Lesions}

Of 1438 oral mucosal lesions detected in the screened population, 777 (54\%) were clinically diagnosed as benign and no patients with any of these 23 different classifications of lesions were referred (Figure 1 and Table 1). This implies the clinicians can readily exclude malignancy even if dietary or cultural risk factors are associated with these Visually Benign Lesions (VBLs). Of the 
$661(46 \%)$ lesions, representing 9 different clinical diagnoses, that were not definitively benign on visual screening, only $66(10 \%)$ were referred as suspected malignancy. The large number of clinically 'not-benign but also not definitively malignancy' OMLs could be termed Visually Complex Lesions (VCLs) to distinguish them from the 'visually benign lesions' and also from the recognised PMDs or OSCC diagnosed after histological evaluation. The VCLs are a heterogenous group with $100 \%$ of erythroleukoplakias, erythroplakias and ulcers suspected to be OSCC referred to the tertiary referral centre. $93 \%$ of observed leukoplakias were referred due to the presence of significant risk factors such as tobacco and paan usage with the remainder detected in low risk individuals who had a regular dentist and deemed inappropriate for a suspected cancer pathway. $67 \%$ of lichenoid reactions and $12 \%$ of reticular LP were also referred but always when present in conjunction with other lesions more indicative of OSCC, such as erythroleukoplakia. 6 out of 7 patients with OSF were referred because of the presence of associated lesions of concern and the other was also already under the care of the hospital. Paan staining itself was not an indication for referral, as it is endemic in areca nut extract users, but $11 \%$ of these individuals also had other referable lesions.

\section{Intraoral Site of Mucosal Lesions}

Mucosal lesions were detected throughout the oral cavity (Table 4) with the largest proportion (48\%) on the buccal mucosa including the buccal sulcus. The buccal area was also the largest single site for the VCLs (36\%) and referred (35\%) OMLs. The buccal mucosa is also often the site of frictional 
keratosis related to the occlusion and therefore was also the single commonest site for VBLs. There were no VBLs observed on the FOM or ventral tongue, which are conventionally high-risk sites for OSCC. 


\section{DISCUSSION}

Oral cancer screening trials often report significant numbers of false positive results, which are unnecessarily worrying for patients as well as an inefficient use of limited healthcare resources. Our data suggests clinicians struggle to accurately define OMLs as benign, or not, because a substantial proportion of benign OMLs share the same clinical features and risk factors as OSCC.

\section{The Value of Classifying OMLs as Visually Complex or Benign}

OMLs of various diagnosis have conventionally been grouped in the literature as benign lesions or PMDs or OSCC. PMDs have a broad definition of "all clinical presentations that carry a risk of cancer" ${ }^{4}$. Discussions are often limited to leukoplakia and erythroplakia such as in the WHO group review by

Napier et. al. ${ }^{7}$ although the broader description may be more helpful clinically, especially in high-risk population where non-leukoplakia PMDs, such as OSF, are more common..

Of 1438 oral mucosal lesions detected in the screened population $54 \%$ were clinically diagnosed as benign and no patients with these lesions were referred (Figure 1). This implies the clinicians could easily exclude malignancy which is consistent with the high specificity reported for direct visual assessment for OSCC $^{10}$. Of the $46 \%$ of lesions that were not definitively benign only a small proportion (10\%) were referred as suspected malignancy. In our study, the number of clinically 'not-benign but also not definitively malignancy' OMLs because at least one of each was referred as suspected cancer, which also are not usually described as PMDs, indicates the need for 
another term such as the Visually Complex Lesion. An important example of an OML that falls between the existing classifications is paan staining whereby its presence alone indicates risk factors for OSCC but it is not itself a PMD although the staining may obscure malignancy related mucosal changes. Therefore, VCL would be a screening-specific term used in direct visual assessment of the oral mucosa relating to any abnormality that was not definitively benign. VCLs would include PMDs and any other mucosal abnormality where the screening dentist could not assign a benign diagnosis and therefore, in high-risk individuals, could not be certain was not malignancy.

\section{Oral Cancer Risk Factor Correlation with Oral Lesions}

Individuals in the screened population who used paan were 3.43 times $(\mathrm{Cl}$ : 1.18 - 5.31) more likely to have any OMLs than those who did not when controlling for the other risk factors of smoking, alcohol and age (Table 2). Smokers were 2.52 times $(\mathrm{Cl}: 1.02$ - 4.54) more likely to present with OMLs than non-smokers controlling for paan and alcohol usage and age effects which would be expected as smoking is known to induce keratosis of the oral mucosa either by direct effect of heat or chemical irritation. Use of tobacco and areca nut compounds resulting in increasing numbers of OMLs is consistent with evidence that these risk habits are directly related to the presence of leukoplakia as shown by Gupta et. al $^{11}$ in a large scale intervention study where stopping tobacco usage also drastically reduced the incidence of leukoplakia. 
There was no statistically significant relationship with use of alcohol and the presence of all OMLs. An explanation could be the small sample size for alcohol users as only $16.6 \%$ of those screened admitted drinking alcohol in a predominantly Islamic origin population. It is also possible that alcohol use is infrequent and minimal, as no attempt was made to quantify units or regularity of use. Alternatively alcohol use may not result in visible OMLs in the same way as paan or smoking as there is no frictional or heat component. Age has previously been linked with the presence of OMLs in the Bangladeshi population ${ }^{5}$ yet our multiple logistic regression analysis showed no association (Table 2). The lack of a relationship in the screened population maybe related to the screened population's relatively low mean age 41.4 (SD 15.9 years). This would imply relatively fewer older people and the resultant small sample size would directly affect the ability to show a statistically significant relationship with the older age ranges. It is also known that logistic regression algorithms are systematically inaccurate for odds ratios when the sample size is less than 500 although conversely the degree of inaccuracy is often much lower than the standard error of the estimate and therefore potentially insignificant ${ }^{12}$. The other possibility is that age presents as a compound risk with increasing time of the other behavioural risk factors and the multiple logistic regression analysis methodology controls for the other risk factors thereby negating this effect. To evaluate this, a univariate linear regression for one continuous exposure variable was carried out on the 1111 screened individuals with the single outcome variable being the presence of any OMLs and no controls for any of the other risk factors (Table 3). The interpretation of the linear regression analysis would be that for every one 
year increase in age, the odds of developing a OMLs increase by a factor of $1.01(95 \% \mathrm{Cl}: 1.00-1.03)$ or by about $1 \%$ each year. The relationship shown by linear regression but not by multiple regression controlling for paan usage and smoking, suggests age presents a cumulative effect of the other risk factors, but in itself is not a risk factor for the development of OMLs. Older patients essentially have more time for pathological features to develop from paan usage and smoking and the incidence of OMLs rises because of an inability to repair this mucosal damage.

For VBLs where the dentist is clinically certain there can be no malignancy there were no statistically significant relationships between risk factors and the presence of OMLs. Conversely, VCLs show a strong relationship with OSCC risk factors.

\section{Significance of the Multiple OMLs}

It has been reported in previous studies of the Bangladeshi population of Tower Hamlets that patients often present with multiple OMLs ${ }^{5}$ but the significance of this has not been evaluated. Our screening dentists also qualitatively reported difficulties in diagnosing patients with OMLs due to the presence of multiple mucosal abnormalities. Therefore, it would be expected to see more patients in the referred population with multiple OMLs than the general screened population. The proportions of patients presenting with 1 OML or multiple OMLs are significantly different between the referred group and the total group of people with OMLs (Figure 2). Comparison of proportions of patients with multiple OMLs shows the composition of the 
referred patient's group is also different to the group of patient's with VBLs but similar to the VCL group. This could simply be because the referred patients, by definition, are a sub-group of VCLs but as they only comprise $10 \%$ of this group, the indication is of some relationship between multiple lesions and referral. Any relationship is complicated by the fact that any one lesion can initiate a referral, if suspicious enough to the clinician, but it would also seem reasonable that more OMLs would increase the screening clinician's level of uncertainty and concern, especially if the lesions are not obviously benign. 


\section{CONCLUSION}

Dentists are often presented with OMLs and specifically need to determine whether these lesions may be or become cancer. Accuracy of this clinical decision making process is extremely variable and if inappropriate may lead to over treatment or a missed opportunity to catch cancer early. Our data suggests that OMLs are highly prevalent in patients displaying risk factors for OSCC making this dilemma even more difficult. We have identified a significant proportion of OMLs, which do not fit the conventional PMD or OSCC classification but are also not definitively benign in their clinical presentation. We have shown that these VCLs, in general have the same risk factors as their OSCC subgroup, although the majority are entirely benign in nature. VCLs can however be distinguished from VBLs by their clinical presentation alone.

VCLs are likely to be the reason for the variability in sensitivity reported in the literature for clinical examination in detecting OSCC. Before oral cancer screening trials can be endorsed, further research is needed to identify clinical, aetiological or molecular markers to differentiate between the subgroups of VCLs (benign OMLs, PMDs and OSCC) that have indistinguishable clinical presentations in patients at high-risk of oral cancer. 


\section{ACKNOWLEDGMENTS}

We are grateful to colleagues who have assisted with this project and acknowledge the contribution of Judith Jones, Ray Croucher, Shamsia Begum, Dipul Gosh and Ryan Salucideen, Barts and the London School of Medicine and Dentistry; Sara Hiom, Jessica Harris and Zoe Norridge, Cancer Research UK. We also acknowledge specific financial support to AW from Rosetrees Trust and Barts Charity, and to AL from the Royal College of Surgeons of England, Faculty of Dental Surgery.

\section{CONSENT AND ETHICS}

Data collection for this study was approved by the East London and The City Research Ethics Committee (08/H0701/98). To permit anonymity, initially all participants provided only verbal and implied consent before being screened. If agreed, written consent was obtained for recording of patient related data but it was made clear that refusing to participate in the data collection exercise did not prevent patients from being screened or referred and no attempt was to be made to elicit why. Throughout the screening activity no participants refused data collection. 


\section{REFERENCES}

1. Walsh T, Liu JL, Brocklehurst P, Glenny AM, Lingen M, Kerr AR, et al. Clinical assessment to screen for the detection of oral cavity cancer and potentially malignant disorders in apparently healthy adults. The Cochrane database of systematic reviews. 2013:CD010173.

2. Brocklehurst PR, Baker SR, Speight PM. Factors affecting the referral of potentially malignant lesions from primary dental care: a pilot study in South Yorkshire. Prim Dent Care. 2009;16:13-8.

3. Nunn H, Lalli A, Fortune F, Croucher R. Oral cancer screening in the Bangladeshi community of Tower Hamlets: a social model. British journal of cancer. 2009;101 Suppl 2:S68-72.

4. Warnakulasuriya S, Johnson NW, van der Waal I. Nomenclature and classification of potentially malignant disorders of the oral mucosa. J Oral Pathol Med. 2007;36:575-80.

5. Pearson N, Croucher R, Marcenes W, O'Farrell M. Prevalence of oral lesions among a sample of Bangladeshi medical users aged 40 years and over living in Tower Hamlets, UK. Int Dent J. 2001;51:30-4.

6. Cawson RA, Odell EW. Cawson's Essentials of Oral Pathology and Oral Medicine. 7th ed: Churchill Livingstone; 2002.

7. Napier SS, Speight PM. Natural history of potentially malignant oral lesions and conditions: an overview of the literature. J Oral Pathol Med. 2008;37:110.

8. Byakodi R, Shipurkar A, Byakodi S, Marathe K. Prevalence of oral soft tissue lesions in Sangli, India. J Community Health. 2011;36:756-9.

9. Saraswathi TR, Ranganathan K, Shanmugam S, Sowmya R, Narasimhan PD, Gunaseelan R. Prevalence of oral lesions in relation to habits: Cross-sectional study in South India. Indian J Dent Res. 2006;17:121-5.

10. Downer MC, Moles DR, Palmer S, Speight PM. A systematic review of measures of effectiveness in screening for oral cancer and precancer. Oral oncology. 2006;42:551-60.

11. Gupta PC, Mehta FS, Pindborg JJ, Bhonsle RB, Murti PR, Daftary DK, et al. Primary prevention trial of oral cancer in india: a 10-year follow-up study. J Oral Pathol Med. 1992;21:433-9.

12. Nemes S, Jonasson JM, Genell A, Steineck G. Bias in odds ratios by logistic regression modelling and sample size. BMC Med Res Methodol. 2009;9:56. 